\title{
In Silico Knockout Screening of Plasmodium falciparum Reactions and Prediction of Novel Essential Reactions by Analysing the Metabolic Network
}

\author{
Jelili Oyelade $\mathbb{D}^{1,2}$ Itunuoluwa Isewon, ${ }^{1,2}$ Efosa Uwoghiren $\mathbb{D}^{1,}, 2$ \\ Olufemi Aromolaran, ${ }^{1,2}$ and Olufunke Oladipupo ${ }^{1}$ \\ ${ }^{1}$ Department of Computer and Information Science, Covenant University, Ota, Ogun State, Nigeria \\ ${ }^{2}$ Covenant University Bioinformatics Research (CUBRe), Covenant University, Ota, Ogun State, Nigeria \\ Correspondence should be addressed to Jelili Oyelade; ola.oyelade@covenantuniversity.edu.ng
}

Received 3 November 2017; Revised 4 February 2018; Accepted 19 February 2018; Published 29 March 2018

Academic Editor: Edmond J. Remarque

Copyright (c) 2018 Jelili Oyelade et al. This is an open access article distributed under the Creative Commons Attribution License, which permits unrestricted use, distribution, and reproduction in any medium, provided the original work is properly cited.

Malaria is an infectious disease that affects close to half a million individuals every year and Plasmodium falciparum is a major cause of malaria. The treatment of this disease could be done effectively if the essential enzymes of this parasite are specifically targeted. Nevertheless, the development of the parasite in resisting existing drugs now makes discovering new drugs a core responsibility. In this study, a novel computational model that makes the prediction of new and validated antimalarial drug target cheaper, easier, and faster has been developed. We have identified new essential reactions as potential targets for drugs in the metabolic network of the parasite. Among the top seven (7) predicted essential reactions, four (4) have been previously identified in earlier studies with biological evidence and one (1) has been with computational evidence. The results from our study were compared with an extensive list of seventy-seven (77) essential reactions with biological evidence from a previous study. We present a list of thirty-one (31) potential candidates for drug targets in Plasmodium falciparum which includes twenty-four (24) new potential candidates for drug targets.

\section{Introduction}

Plasmodium falciparum, a leading cause of malaria, has a complex life cycle $[1,2]$. The female mosquito of the Anopheles genre is responsible for all the transference of malaria from one patient to another $[1,2]$. Despite the colossal efforts put in to fight malaria, the disease still affects up to over 200 million people every year with close to half a million dying [15]. The Plasmodium falciparum lifecycle comprises three (3) important developmental stages: the mosquito stage, the liver stage, and the blood stage [6]. Sporozoites injected into a host by a mosquito that is infected travel to the liver and begin the hepatic stage of the life cycle of the Plasmodium by invading hepatocytes. Here, they get to increase and segregate into schizonts, then comprising numerous hepatic merozoites. All of these merozoites are successively set loose into the blood where the erythrocytic stage is initiated and begins by invading and duplicating inside the red blood cells (RBCs) $[1,7]$.

Evidences abound to the fact that the parasite is already developing resistance to many front-line antimalaria therapies. Therefore, novel antimalaria cures are in immediate need to combat the drug-resistant malaria parasite $[5,8]$. The metabolism of Plasmodium falciparum (P. f.) in cells that are infected would be quite a potential source of targets for novel drugs but it is complex and difficult to understand intuitively. In silico methods can handle and take care of this complexity. They also give room for integrative analyses of the cell metabolism [9]. In silico methods play an important role in the identification and prediction of new drugs [10] and facilitate the prospects for the discovery of imminent drug leads [11]. They have been successfully used to predict potential drug targets, alter already existing proteins so as to have an improved stability and functionality, and reduce the 
search space for drug prediction $[5,8,12]$. The challenge of $P$. $f$ 's resistance to most identified antimalarial drugs has given rise to the increase of antimalarial drug discovery research $[4,13,14]$. Hence, research on the development of novel drug targets which would serve as an effective solution for malaria treatment is urgently needed [3,13-15]. Due to upgrades of $P$. $f$. genome, the reconstruction of the metabolic network is required for a comprehensive understanding of the molecular mechanisms of the organism $[8,16,17]$. Though experimental validation of novel drug target by reaction knockout methods could be accurate, they are time consuming and are a major impediment towards discovery of drug targets [5]. Thus, this study provides a novel computational model that predicts essential reactions and makes the validation of predicted antimalarial drug target cheaper, easier, and faster. It also gives a deeper understanding of the metabolic activities of $P$. $f$. This study identifies new reactions as potential targets for drugs in the metabolic network of $P$. $f$. that contributes to its survival in the host and validates predicted drug targets. The computational model used in this paper is an enhancement of the computational model used in our preceding paper [18]. The computational model used enhances the results and enables computational analysis of large dataset.

The malaria parasite metabolic pathways are quite different from those of its human host. This uniqueness can be exploited in the design of therapeutic strategies [1921]. Metabolic pathways are chains of connected enzymatic reactions that take place inside a cell $[22,23]$. They form a different chemical compound by modifying a principal compound which is then passed on to start an alternative pathway, used up or kept by the cell [24]. The representation of a metabolic pathway is generally a graphical network of chemical reactions [24]. The stoichiometry represents the quantifiable relations amid reactants and products in a balanced chemical reaction. Combinations of information from different sources such as genomics, network analysis and simulation, and biochemistry are necessary in the study of a metabolic pathway [25]. More than one metabolic pathway which consists of a chain of reactions that contribute to the synthesis or degradation of the same metabolite makes up a metabolic network. Diverse data sources guide the genomescale reconstruction of metabolic networks [5]. Marwan et al. [26] regarded metabolic networks as a flow of substance from side to side of biochemical intermediates that are converted into each other. A metabolic network is simply a graphical representation of metabolism [27], characterized by a flow of substance through biochemical intermediates that are interconverted into each other [26]. Metabolic networks are useful tools for deepening our understanding of the metabolism and the role of genes through the evaluation of gene essentiality [28]. Therefore, a metabolic network is simply a diagrammatic illustration of the chemical processes that occur in maintaining the living state of the cells and the organism [27]. KEGG (Kyoto Encyclopaedia of Genes and Genomes) is an integrated primary database resource that consists of 16 main databases for biological interpretation of high-throughput data which are characterized as chemical, systems, genomic, and health information and genome sequences [29]. KEGG is a database resource for comprehending higher order functions and utilities that are comprised in the biological system [30-32]. MetaCyc is a general database consisting of enzymes and metabolic pathways. MetaCyc acts in the capacity of a reference database of small-molecule metabolism which is not redundant and is comprised of metabolic pathways that are experimentally verified and enzyme information selected from the different scientific literature. It makes a unique resource of high quality available for metabolic pathways and enzymes because it comprises only experimentally explained knowledge [3335]. MetaCyc is one of the major collections of metabolic pathways with over 1700 pathways [34].

1.1. Essentiality of a Reaction in a Metabolic Network. Identifying essential reactions in a metabolic network allows the identification of potential drug targets in the network $[36,37]$. Essential reactions are widely recognized as ideal drug target candidates since deleting them could lead to a compromise of integrity of the network [37,38]. Essential reactions are those reactions of an organism that are thought to be critical for its survival because without them the network cannot function [39]. The predictions of essential reactions experimentally even though largely accurate have a need for substantial time and resources, even for organisms that are well-studied, and they are not at all times practical [5, 37, 40], while the predictions of essential reactions computationally are faster and quite less expensive and they have the capability to decrease the search space for new targets for drugs in a metabolic network which can then be validated experimentally $[5,37]$. Deleting just one essential reaction is enough to cause lethality or infertility in the network. In comparison to nonessential reactions, essential reactions are expected to be more preserved in biological evolution $[36,37]$. The essentiality of a node in a network is explained in Figure 1 which depicts a network with three different essentiality levels, the red node being the most essential node followed by the blue node in the network and the yellow nodes are the least essential nodes in the network. For example, if the hub node (the red node) is knocked out, it will affect the entire network in the system.

1.2. Methods of Detecting Essentiality of Reactions. Flux balance analysis (FBA) is an in silico method used in gaining deepened understanding into the abilities and the metabolic behaviour of a cell $[5,42,43]$. It is an extensively used and deep-rooted method to assess the essential genes of a particular organism. FBA is used extensively in the study of reconstructing the metabolic network of a genome based on mass conservation. Flux balance analysis envisages the complete growth rate of a particular organism or rate of utilization of any particular metabolite by simply calculating how the metabolites flow through the metabolic network [43]. The stoichiometry information of the metabolic network along with the metabolic target functions essential to the cell of interest is also necessitated by FBA $[5,43]$. However, there are quite a number of baffling failures of FBA techniques in predicting the essentiality of a gene in a particular organism [44-46]. FBA suffers from incomplete annotation of the proteins in a genome [46]; FBA suffers 


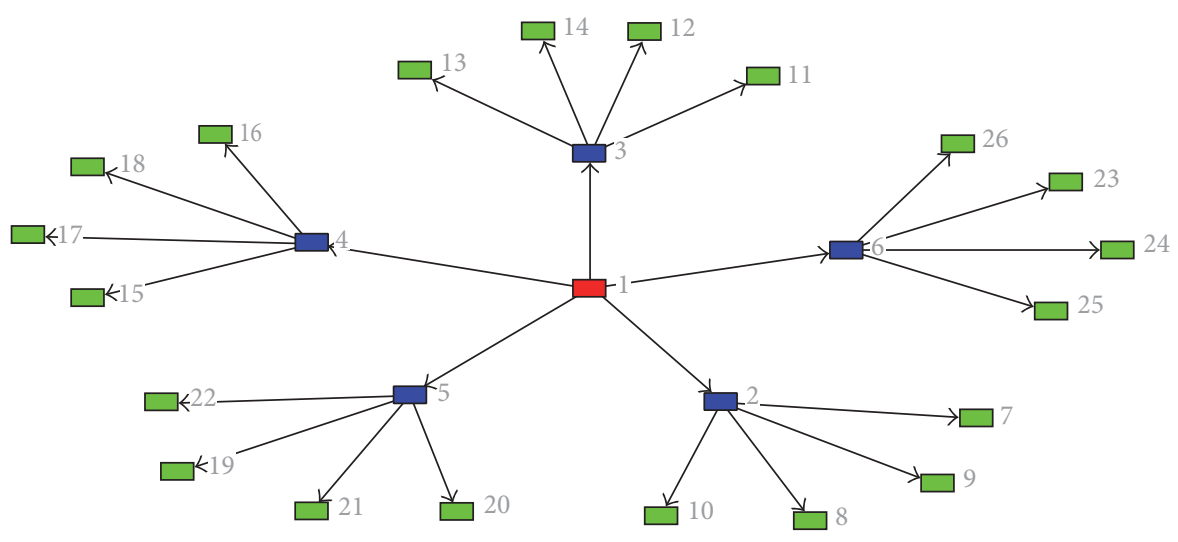

Figure 1: A network tree which paints the essentiality of each node to the survival of the tree [41].

greatly in defining biologically relevant objective function [46]. The information about the stoichiometry of the reaction pathway is required when using FBA [43]; FBA technique fails to moderately correlate between evolutionary rate and predicted gene dispensability [44, 45]; FBA approach is in need of perfect specification that defines the production of biomass and the nutrition that is available under explicitly given environmental conditions [47]. Minimal Metabolic Behaviours [48] can be seen as mathematical method to approach metabolic pathway analysis; it makes use of the outer description of the steady-state flux cone, which is determined by the sets or number of nonnegativity constraints [48]. When compared to already existing methods, its description is more compacted. It proposes an integrated method to the studying of the metabolic networks [48]. Elementary mode analysis (EMA) is a veritable metabolic pathway tool that considers stoichiometric and thermodynamics when evaluating whether a particular metabolic route or network is feasible and likely for a set of proteins/enzymes [49]. This method is valuable for the purpose of decomposing the intricate metabolic network made up of highly interconnected reactions into uniquely organized pathways. Elementary mode analysis is a tool used to identify the structure of a metabolic network that connects the cellular phenotype to the corresponding genotype. Elementary modes increase rapidly with regard to the network size and because of this the time to compute the network increases largely with respect to the size of the network thereby limiting analysis to pathways and not the entire metabolic network of a genome $[50,51]$. EMA is based mainly on the reaction equations stoichiometry and the steady-state conditions of the particular organism [52]. When using metabolic flux analysis (MFA) $[53,54]$, any change made in the metabolic pathway fluxes is measured. Information like this gives more insights into how the metabolic pathways are being regulated and could likely suggest novel targets for added metabolic engineering of the strains [55]. Metabolic flux analysis (MFA) denotes an influential tool for systems biology research [56]. A major setback of metabolic flux analysis for a lot of biological systems is however that the amount of constraint is often not sufficient to observe all essential intracellular metabolic pathways $[55,57]$. In load point and choke point analysis, the number of $k$-shortest paths passing through metabolites and its closest neighbour links is defined as the load point of a particular metabolite in a metabolic network. The usefulness or the importance of a particular metabolite in the metabolic network of an organism is determined by load points and choke points [58-60]. The choke points are ordered by the amount of $k$-shortest paths passing through them. When a choke point in an organism is absent, the organism can rarely survive $[59,60]$. Thermodynamics-based Flux Analysis (TFA) is a variant of metabolic flux analysis presented with the capacity of producing thermodynamically feasible flux and metabolite movement profiles on a genome scale $[61,62]$. TMFA includes the utilization of an arrangement of straight thermodynamic constraints notwithstanding the mass balance limitations ordinarily utilized as a part of MFA [9, 62]. TMFA produces flux circulations not encompassing any form of thermodynamically infeasible responses or pathways, and in addition to reaction fluxes it makes lots of information about the range of a substance formed or necessary for metabolism activities and the free energy change of reactions available $[9,61,62]$. Metabolic Control Analysis helps in determining quantitatively the level or amount of influence that different enzymes have in the intracellular network on very important flux (or function) [63]. MCA is categorized as a postgenomic device used in comprehending the principles that govern a metabolic network which is disseminated among numerous enzymatic steps [64]. MCA studies provide rational and quantitative criteria to select enzymes for drug target development [65]. The application of Metabolic Control Analysis makes it possible to recognize the group of proteins that necessarily have to be altered to achieve an effective modulation of the intracellular networks of biotechnological or clinical relevance [63].

1.3. Reaction Deletion/Perturbation Studies. Perturbation is an approach generally applied to study the conduct and atomic components underlying cellular systems [66]. A perturbation can likewise be focused on the interruption of a specific cell segment, for instance, by deleting reactions or by RNA-intervened knockdown. These two universal types 
of perturbation are frequently used [66]. Perturbation in a particular pathway happens by interfering with the flow of the signal of a given network which gives knowledge into both their arrangement and their downstream targets. To begin with, with the interference at a specific node in the pathway, the signal cannot be conveyed further. Secondly, every node in the pathway may have its own (immediate or aberrant) commitment to the perturbation impacts, for example, reaction expression changes [67].

In this study, the essentiality of the different reactions was determined. Therefore, a list of indispensable reactions in the Plasmodium falciparum metabolic network was identified and proposed as potential drug target for Plasmodium falciparum.

\section{Materials and Methods}

2.1. Reconstruction of the Metabolic Network. In this study two different resources were considered for the reconstruction of the metabolic network, which is the genome-scale metabolic dataset of the 3D7 strain of Plasmodium falciparum which was extracted from the BIOCYC flat file database version 19.5 [68] because of its comprehensiveness and robustness where the dataset contained 894 metabolic reactions and these reactions were catalyzed by a total number of 710 enzymes; also the metabolic dataset of the genome-scale 3D7 strain of Plasmodium falciparum from [9] was extracted to fill the gaps in the BIOCYC genome-scale metabolic dataset of which the dataset contained 670 metabolic reactions and these reactions were catalyzed by a total number of 325 enzymes. The raw data used by Chiappino-Pepe includes the protein FASTA files version 11.1 with protein sequences for P. falciparum 3D7 from PlasmoDB and the version of KEGG as of July 2014. The BIOCYC identifiers were chosen for this study as the generally accepted means of identification. Plasmodium falciparum reactions gotten from Chiappino-Pepe were mapped to BIOCYC reactions via enzymes commission numbers and common name. The reconstructed metabolic network of Plasmodium falciparum is made available as SBML file with some reactions considered to be reversible and some considered to be irreversible. In this study, currency metabolites of the 24 currency metabolites outlined by [69] were removed from the reconstructed genome-scale metabolic dataset.

2.2. In Silico Knockout Analysis. Once the metabolic network was reconstructed, a Plasmodium falciparum metabolic network was created leading us to perform an in silico knockout experiment and analysis on the metabolic network enabling us to analyse the network for the essentiality and perturbation of the knocked-out reactions and we also moved further to determine the perturbations and essentiality of all reactions in the network. When a transition (reaction) is knocked out, all reactions that have a corresponding reactant or product of the knocked-out reaction are equally knocked out, helping to ascertain the effect of that reaction to the network. Single knockout analyses were performed and our results were outputted in .txt formats to list the reactions that were affected after knocking out a specific reaction.
2.3. The Algorithm. In the network a reaction is knocked out to determine the dependent reaction on the knocked-out reaction. The procedure for determining which reaction is connected to the knockout is outlined in the following steps.

Step 1. Start.

Step 2. Get the SBML file.

Step 3. Extract all reactions in the file.

Step 4. Extract all products and reactants related to the various reactions.

Step 5. Identify reaction to knockout initially.

Step 6. Get other reactions that are linked to the identified reaction which can be knocked out.

Step 7. Determine if the other reactions are linked to other reactions.

Step 8. If true, do not knock out the reaction; else knock out the reaction.

Step 9. Repeat Steps 6-8 for the resulting reactions.

Step 10. If there are no other reactions to be knocked out, attach the resulting reactions to the initial reaction.

Step 11. Assign "knocked-out reactions" as the list of resulting reactions attached to the initial knocked-out reaction.

Step 12. Repeat Steps 5-11 to get all results for every reaction in the network.

Step 13. Determine the essentiality of each reaction by comparing the knocked-out items to the total network.

Step 14. Determine the most essential reactions by comparing the results of Step 13.

Step 15. Determine the least essential reactions by comparing the results of Step 13.

Step 16. Extract result to a spreadsheet file.

Step 17. Make visualisation of the result in the spreadsheet file.

Step 18. Stop.

All above steps are performed for all reactions in the network enabling this study to predict the essentiality of all reactions in the network and exporting them in hierarchical order.

$$
\begin{gathered}
\text { If } R 1=\mathrm{SR} \\
\mathrm{Kc} \forall R \\
\text { If } R(\mathrm{Re})=R 1(\mathrm{Pr}) \\
\text { End }
\end{gathered}
$$




\author{
IF $R 1=S R$ \\ $\mathrm{Kc} \forall R$ \\ If $R(\operatorname{Pr})=R 1(\mathrm{Re})$ \\ End \\ IF $R 1(\operatorname{Re})=R(\operatorname{Pr})$ \\ DKc $R$ \\ End

IF $R 1(\operatorname{Pr})=R(\mathrm{Re})$
DKc $R$
End

$\mathrm{R}$ represents reaction, $\mathrm{SR}$ represents selected reaction, Re represents reactant, Pr represents product, Kc means knockout, and DKc means no knockout.

In this study reconstructed network, we determined the essentiality of every reaction in the network and proposed some essential reactions and validated the essentiality of previous proposed reactions in literature. The essentiality of all reactions is saved in a txt file for easy access. The formula for determining the essentiality of every reaction in the network is outlined in the following steps:

$$
\begin{aligned}
r i & =r 1+r 2+r 3+\cdots+r n \\
E(r) & =\frac{\sum k(r i)}{\sum N(r i)} \% .
\end{aligned}
$$

$r i$ represents the list of reactions, $E(r)$ represents the essentiality of a reaction in the reconstructed metabolic network, $k(r i)$ represents the knocked-out reactions in the reconstructed metabolic network, $N(r i)$ represents a reaction in the network, $\sum k(r i)$ represents the summation of the knockedout reactions in the reconstructed metabolic network, and $\sum N(r i)$ represents the summation of all reactions in the network.

2.4. The Gold Standard. An extensive list of 77 essential reactions in Plasmodium falciparum which mostly have been predicted in several literatures to be druggable was considered when testing and validating our model and these 77 essential reactions are given in the supplement (supplementary Table S1). A large percentage of this gold standard was considered in our network and validated by our model as essential. Our network was constructed as a directed-bipartite graph with two different types of nodes.

\section{Results and Discussion}

3.1. Results. Computationally predicted essential reactions from six different literatures were compared with our method. Ten reactions that were common to over $80 \%$ of all literature considered in this study were identified and validated to be essential by our method, thereby confirming our method as valuable computational technique for validation of predicted drug target as given in Table 1, respectively. The network of Plasmodium falciparum used in our study was analysed and each reaction in the network was knocked out and the essentiality of each reaction in the network was determined. The top seven (7) predicted most essential reactions based on our method in the network are represented in Table 2, four (4) of which were identified to be found in the gold standards including superoxide dismutase, 3-phosphoshikimate 1-carboxyvinyltransferase, 5-O(1-carboxyvinyl)-3-phosphoshikimate phosphate-lyase, and adenosylhomocysteinase and one (1) was already predicted as essential computationally which is methionine adenosyltransferase. The reactions are well represented by BIOCYC database reaction unique identification number. The reactions that are represented boldly are reactions that are represented in gold standards.

3.1.1. Knocked-Out Reactions for the Most Essential Reactions. The reactions that were knocked out from the network when the top seven (7) most essential reactions from our network were knocked out are given in the Supplementary Table S2(a-g). Table S2(a) represents the reactions that were knocked out by reaction SUPEROX-DISMUT-RXN which is responsible for knocking out 94 other reactions in the metabolic network. Table S2(b) represents the reactions that were knocked out by reaction CATAL-RXN of which is responsible for knocking out another 74 reactions in the network. In Table S2(c-g), reactions S-ADENMETSYNRXN, 2.5.1.19-RXN, CHORISMATE-SYNTHASE-RXN, SHIKIMATE-KINASE-RXN, and ADENOSYLHOMOCYSTEINASE-RXN, respectively, were responsible for knocking out another 49,48,48, 48, and 39 reactions in the metabolic network. Table S2(d) represents the reactions that were knocked out by reaction 2.5.1.19-RXN which is responsible for knocking out another 48 reactions in the metabolic network used.

The predicted essential reactions based on the analysis of our network are given in the Supplementary Table S3 and Figures S1 and S2. Table S3 lists all reactions that are seemingly essential to the network used in this study and their essentiality level where these reactions were compared with gold standards and computationally predicted reactions. This study finally presents a polished list of 31 potential candidates for drug targets in Plasmodium falciparum which includes 24 new potential candidates for drug targets of which 9 are orphans and 7 potential candidates for drug target which has been predicted computationally in literature of which 1 is an orphan. The reactions are presented in Table 3.

3.2. Discussion. This study established a novel method that performed an analysis on the genome-scale metabolic network of Plasmodium falciparum and identifies reactions in the network that are essential to the survival of the network according to its essentiality. These essential reactions are predicted as potential drug targets for Plasmodium falciparum; the essentiality of the reaction in the network is listed according to its effect on the network when knocked out (the 


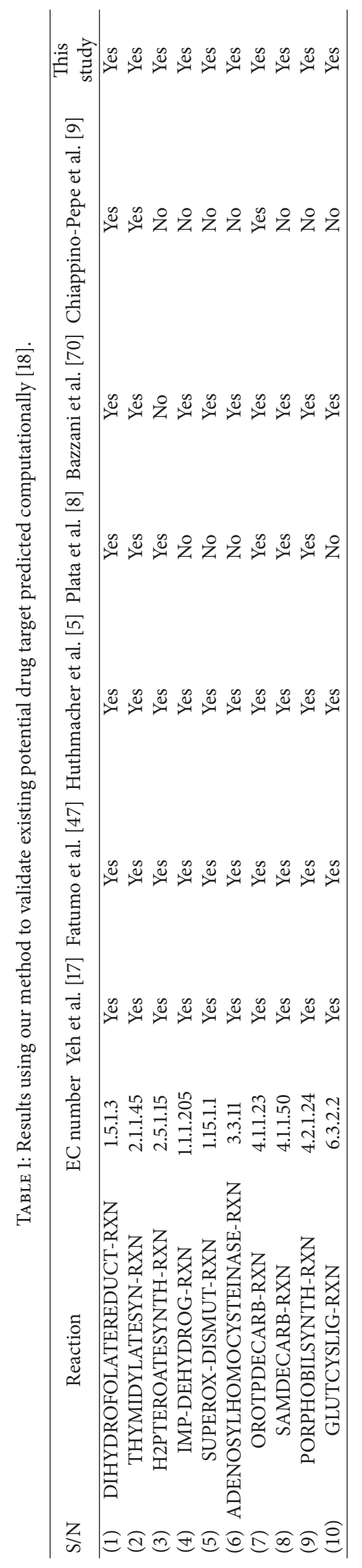




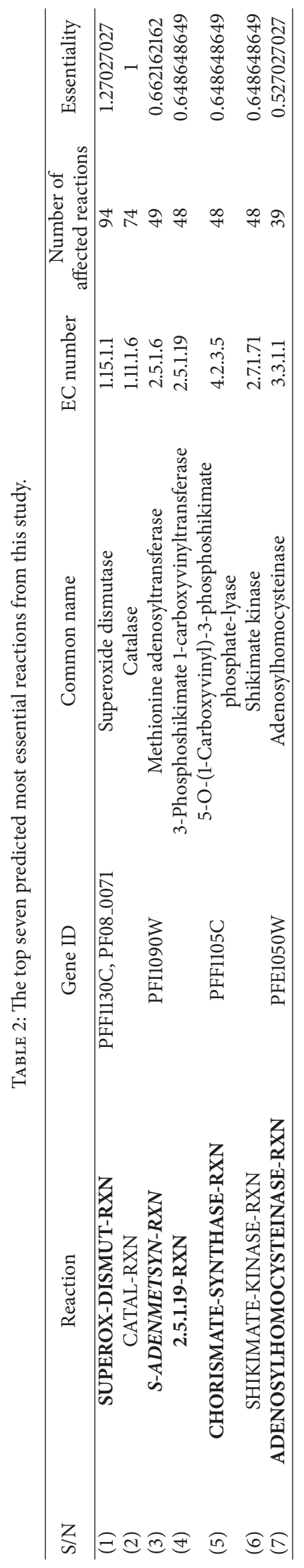




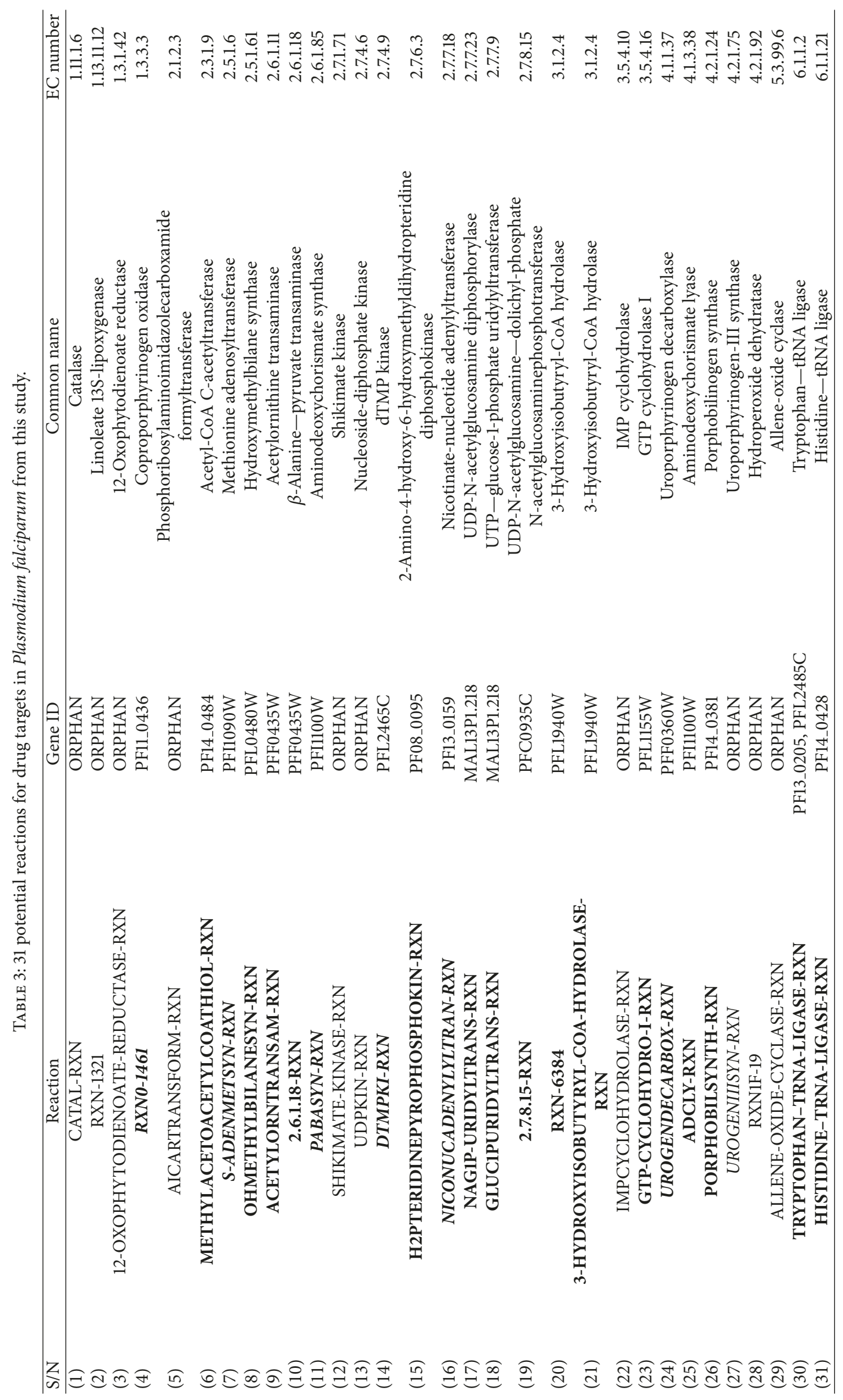


amount of reactions knocked out when the parent reaction is knocked out). This study identifies that there are over 200 essential reactions in the network of which, among the top 7 predicted most essential reactions, 4 were identified to be found in the gold standard which includes superoxide dismutase, 3-phosphoshikimate 1-carboxyvinyltransferase, 5-O-(1-carboxyvinyl)-3-phosphoshikimate phosphate-lyase, and adenosylhomocysteinase and 1 was already predicted as essential computationally which is methionine adenosyltransferase. The result of our study was compared with an extensive list of 77 essential reactions with biological evidence. We finally present a polished list of 31 including 24 new potential candidates for drug targets of which 9 are orphans and 7 potential candidates for drug target which has been predicted computationally in literature of which 1 is an orphan. This model also helps to improve the understanding of the biological processes within this network and any other metabolic network. It would be quite exciting to further our research by confirming our in silico predictions experimentally and also test if our essential reactions can be successfully targeted without collateral partial or complete targeting of the corresponding human reactions. Potential candidates for drug targets for Plasmodium falciparum already biologically proven were disregarded from our list as our method validated a number of them. The method developed could handle multiple knockouts but we plan to do this when we have some amount of drug combination to validate our method. The method used in this study is capable of predicting essential reaction in any other organisms of a robust genome-scale metabolic network.

\section{Conclusion}

The dominance of malaria in resistance to identified antimalarial drugs in current circulation has given rise to the increase of antimalarial drug discovery research. Hence, researches on the development of novel drug targets which would serve as effective solutions for malaria treatment are urgently needed.

In this study, a novel computational model was constructed which makes the validation of predicted antimalarial drug target cheaper, easier, and faster as well as the validation of $P$. $f$. metabolic reactions under different growth conditions and perturbations. We have been able to identify new essential reactions as possible targets for drugs in the metabolic network of $P$. $f$. that contributes to its survival in the host and validate predicted drug targets. The computational model used in this study enhances and enables computational analysis of large dataset.

Generally, the results from this study make a deep understanding of the metabolism of $P$. $f$. available and provide guidance to experimental studies helping to develop a better description of $P$. falciparum metabolism and to identify antimalarial drug targets.

\section{Data Availability}

The datasets used in this study are available in http:// bioinformatics.ai.sri.com/ecocyc/dist/flatfiles-52983746/.

\section{Conflicts of Interest}

The authors disclose no potential conflicts of interest.

\section{Authors' Contributions}

All authors contributed to this work. Jelili Oyelade and Itunuoluwa Isewon contributed to original idea and conception. Implementation of the concept was achieved by Efosa Uwoghiren. Jelili Oyelade, Itunuoluwa Isewon, Efosa Uwoghiren, Olufemi Aromolaran, and Olufunke Oladipupo wrote the manuscript. Jelili Oyelade, Itunuoluwa Isewon, and Efosa Uwoghiren were responsible for structure and argument for the paper. Jelili Oyelade, Itunuoluwa Isewon, Efosa Uwoghiren, Olufemi Aromolaran, and Olufunke Oladipupo were responsible for critical revision and approved final version.

\section{Acknowledgments}

This work is fully supported by the Covenant University and Covenant University Center for Research, Innovation and Discovery with Grant no. CUCRID RG 034.04.15/FS.

\section{Supplementary Materials}

Appendix A. Table S1: the gold standard. This consists of reactions which are biologically proven to be targets of already accepted/tested drugs. Table S2: knocked-out reactions for reactions a-g. Appendix B. Table S3: all predicted essential reactions in the network used in this study. The predicted essential reactions based on our analysis in the network are presented in this table, most of which were identified to be found in the gold standards or have been predicted to be essential to the network in previous study. Figure S1: graph to show number of reactions considered and essentiality of reactions. Figure S2: graph to represent essentiality of reaction. (Supplementary Materials)

\section{References}

[1] K. Modrzynska, C. Pfander, L. Chappell et al., "A Knockout Screen of ApiAP2 Genes Reveals Networks of Interacting Transcriptional Regulators Controlling the Plasmodium Life Cycle," Cell Host \& Microbe, vol. 21, no. 1, pp. 11-22, 2017.

[2] K. Koussis, E. Goulielmaki, A. Chalari et al., "Targeted deletion of a Plasmodium site- 2 protease impairs life cycle progression in the mammalian host," PLoS ONE, vol. 12, no. 1, Article ID e0170260, 2017.

[3] WHO, World Malaria Report 2016, WHO, 2016.

[4] C. Ekenna, S. Fatumo, and E. Adebiyi, "In-silico evaluation of malaria drug targets," International Journal of Engineering and Technology, vol. 2, no. 2, 2010.

[5] C. Huthmacher, A. Hoppe, S. Bulik, and H.-G. Holzhütter, "Antimalarial drug targets in Plasmodium falciparum predicted by stage-specific metabolic network analysis," BMC Systems Biology, vol. 4, article no. 120, 2010.

[6] Z. Bozdech, M. Llinás, B. L. Pulliam, E. D. Wong, J. Zhu, and J. L. DeRisi, "The transcriptome of the intraerythrocytic 
developmental cycle of Plasmodium falciparum," PLoS Biology, vol. 1, article e5, 2003.

[7] V. Soulard, H. Bosson-Vanga, A. Lorthiois et al., "Plasmodium falciparum full life cycle and Plasmodium ovale liver stages in humanized mice," Nature Communications, vol. 6, article no. 7690, 2015.

[8] G. Plata, T.-L. Hsiao, K. L. Olszewski, M. Llinás, and D. Vitkup, "Reconstruction and flux-balance analysis of the Plasmodium falciparum metabolic network," Molecular Systems Biology, vol. 6, article no. 408, 2010.

[9] A. Chiappino-Pepe, S. Tymoshenko, M. Ataman, D. SoldatiFavre, and V. Hatzimanikatis, "Bioenergetics-based modeling of Plasmodium falciparum metabolism reveals its essential genes, nutritional requirements, and thermodynamic bottlenecks," PLoS Computational Biology, vol. 13, no. 3, Article ID e1005397, 2017.

[10] A. Wadood, N. Ahmed, L. Shah, A. Ahmad, H. Hassan, and S. Shams, "In-silico drug design: An approach which revolutionarised the drug discovery process," OA Drug Design and Delivery, vol. 1, no. 1, 2013.

[11] A. V. Le, T. C. Q. Phan, and T. H. Nguyen, "In silico Drug Design: Prospective for Drug Lead Discovery," Int. J. Eng. Sci. Invent, vol. 4, no. 10, pp. 2319-6726, 2015.

[12] J. L. Klepeis and A. C. Floudas, "Floudas, In silico protein design: a combinatorial and global optimization approach," SIAM News, vol. 37, no. 1, 2004.

[13] A. Golldack, B. Henke, B. Bergmann et al., "Substrate-analogous inhibitors exert antimalarial action by targeting the Plasmodium lactate transporter PfFNT at nanomolar scale," PLoS Pathogens, vol. 13, no. 2, Article ID e1006172, 2017.

[14] K. Sahan, C. Pell, F. Smithuis et al., "Community engagement and the social context of targeted malaria treatment: a qualitative study in Kayin (Karen) State, Myanmar," Malaria Journal, vol. 16, no. 1, article no. 75, 2017.

[15] S. V. Hapuarachchi, S. A. Cobbold, S. H. Shafik et al., "The Malaria Parasite's Lactate Transporter PfFNT Is the Target of Antiplasmodial Compounds Identified in Whole Cell Phenotypic Screens," PLoS Pathogens, vol. 13, no. 2, Article ID e1006180, 2017.

[16] C. Francke, R. J. Siezen, and B. Teusink, "Reconstructing the metabolic network of a bacterium from its genome," Trends in Microbiology, vol. 13, no. 11, pp. 550-558, 2005.

[17] I. Yeh, T. Hanekamp, S. Tsoka, P. D. Karp, and R. B. Altman, "Computational analysis of Plasmodium falciparum metabolism: organizing genomic information to facilitate drug discovery," Genome Research, vol. 14, no. 5, pp. 917-924, 2004.

[18] J. Oyelade, E. Uwoghiren, I. Isewon, F. Aromolaran, and O. Oladipupo, "In-silico validation of the essentiality of reactions in plasmodium falciparum metabolic network," in Proceedings of the World Congress on Engineering and Computer Science, vol. 2, 2017.

[19] K. Nakatani, H. Ishikawa, S. Aono, and Y. Mizutani, "Identification of essential histidine residues involved in heme binding and hemozoin formation in heme detoxification protein from plasmodium falciparum," Scientific Reports, vol. 4, article no. 6137, 2014.

[20] E. Hempelmann, "Hemozoin biocrystallization in Plasmodium falciparum and the antimalarial activity of crystallization inhibitors," Parasitology Research, vol. 100, no. 4, pp. 671-676, 2007.

[21] N. Lang-Unnasch and A. D. Murphy, "Metabolic changes of the malaria parasite during the transition from the human to the mosquito host," Annual Review of Microbiology, vol. 52, pp. 561590, 1998.

[22] M. Klapa and G. Stephanopoulos, "Metabolic flux analysis," Bioreact Eng, 2000.

[23] L. Popova-Zeugmann, M. Heiner, and I. Koch, "Time petri nets for modelling and analysis of biochemical networks," Fundamenta Informaticae, vol. 67, no. 1-3, pp. 149-162, 2005.

[24] A. H. Romano and T. Conway, "Evolution of carbohydrate metabolic pathways," Research in Microbiology, vol. 147, no. 67, pp. 448-455, 1996.

[25] P. Baldan, N. Cocco, A. Marin, and M. Simeoni, "Petri nets for modelling metabolic pathways: a survey," Natural Computing, vol. 9, no. 4, pp. 955-989, 2010.

[26] W. Marwan, A. Wagler, and R. Weismantel, "Petri nets as a framework for the reconstruction and analysis of signal transduction pathways and regulatory networks," Natural Computing, vol. 10, no. 2, pp. 639-654, 2011.

[27] V. Lacroix, L. Cottret, P. Thébault, and M.-F. Sagot, "An introduction to metabolic networks and their structural analysis," IEEE Transactions on Computational Biology and Bioinformatics, vol. 5, no. 4, pp. 594-617, 2008.

[28] H.-S. Song, R. S. McClure, H. C. Bernstein, C. C. Overall, E. A. Hill, and A. S. Beliaev, "Integrated in silico analyses of regulatory and metabolic networks of Synechococcus sp. PCC 7002 reveal relationships between gene centrality and essentiality," Life, vol. 5, no. 2, pp. 1127-1140, 2015.

[29] M. Kanehisa, Y. Sato, M. Kawashima, M. Furumichi, and M. Tanabe, "KEGG as a reference resource for gene and protein annotation," Nucleic Acids Research, vol. 44, no. 1, pp. D457D462, 2016.

[30] M. Kanehisa, S. Goto, S. Kawashima, and A. Nakaya, "The KEGG databases at GenomeNet," Nucleic Acids Research, vol. 30, no. 1, pp. 42-46, 2002.

[31] M. Kanehisa, S. Goto, M. Hattori et al., "From genomics to chemical genomics: new developments in KEGG," Nucleic Acids Research, vol. 34, pp. D354-D357, 2006.

[32] M. Kanehisa, S. Goto, M. Furumichi, M. Tanabe, and M. Hirakawa, "KEGG for representation and analysis of molecular networks involving diseases and drugs," Nucleic Acids Research, vol. 38, no. 1, pp. D355-D360, 2009.

[33] R. Caspi, H. Foerster, C. A. Fulcher et al., "The MetaCyc database of metabolic pathways and enzymes and the BioCyc collection of Pathway/Genome databases," Nucleic Acids Research, vol. 36, supplement 1, pp. D623-D631, 2008.

[34] R. Caspi, T. Altman, K. Dreher et al., "The MetaCyc database of metabolic pathways and enzymes and the BioCyc collection of pathway/genome databases," Nucleic Acids Research, vol. 40, no. 1, pp. D742-D753, 2012.

[35] R. Caspi, "MetaCyc: a multiorganism database of metabolic pathways and enzymes," Nucleic Acids Research, vol. 34, no. 90001, pp. D511-D516, 2006.

[36] K. Plaimas, R. Eils, and R. König, "Identifying essential genes in bacterial metabolic networks with machine learning methods," BMC Systems Biology, vol. 4, article no. 56, 2010.

[37] C. Qin, Y. Sun, and Y. Dong, "A new method for identifying essential proteins based on network topology properties and protein complexes," PLoS ONE, vol. 11, no. 8, Article ID e0161042, 2016.

[38] M. Jing, "Metabolic Network Based Gene Essentiality Analysis," 2012. 
[39] J. Oyelade, I. Isewon, S. Rotimi, and I. Okunoren, "Modeling of the glycolysis pathway in plasmodium falciparum using petri nets," Bioinformatics and Biology Insights, vol. 10, pp. 49-57, 2016.

[40] X. Zhang, M. L. Acencio, and N. Lemke, "Corrigendum: Predicting essential genes and proteins based on machine learning and network topological features: A comprehensive review," Frontiers in Physiology, vol. 7, no. 617, 2016.

[41] Y. Gao and G. Madey, "Network Analysis of the SourceForge.net Community," 2006, http://innovate.ucsb.edu/939-yongqin-gaoand-greg-madey-network-analysis-of-the-sourceforge-net-community.

[42] E. P. Gianchandani, A. K. Chavali, and J. A. Papin, "The application of flux balance analysis in systems biology," Wiley Interdisciplinary Reviews: Systems Biology and Medicine, vol. 2, no. 3, pp. 372-382, 2010.

[43] N. Dholakia, P. Dhandhukia, and N. Roy, "Screening of potential targets in Plasmodium falciparum using stage-specific metabolic network analysis," Molecular Diversity, vol. 19, no. 4, pp. 991-1002, 2015.

[44] B. Papp, C. Pál, and L. D. Hurst, "Metabolic network analysis of the causes and evolution of enzyme dispensability in yeast," Nature, vol. 429, no. 6992, pp. 661-664, 2004.

[45] C. Jacobs, L. Lambourne, Y. Xia, and D. Segrè, "Upon accounting for the impact of isoenzyme loss, gene deletion costs anticorrelate with their evolutionary rates," PLOS ONE, vol. 12, no. 1, Article ID e0170164, 2017.

[46] K. Raman and N. Chandra, "Flux balance analysis of biological systems: applications and challenges," Briefings in Bioinformatics, vol. 10, no. 4, pp. 435-449, 2009.

[47] S. Fatumo, K. Plaimas, J.-P. Mallm et al., "Estimating novel potential drug targets of Plasmodium falciparum by analysing the metabolic network of knock-out strains in silico," Infection, Genetics and Evolution, vol. 9, no. 3, pp. 351-358, 2009.

[48] A. Larhlimi and A. Bockmayr, "Minimal direction cuts in metabolic networks," in Proceedings of the 3rd International Symposium on Computational Life Science, CompLife 2007, pp. 73-86, Netherlands, October 2007.

[49] C. T. Trinh, A. Wlaschin, and F. Srienc, "Elementary mode analysis: A useful metabolic pathway analysis tool for characterizing cellular metabolism," Applied Microbiology and Biotechnology, vol. 81, no. 5, pp. 813-826, 2009.

[50] B. A. Boghigian, H. Shi, K. Lee, and B. A. Pfeifer, "Utilizing elementary mode analysis, pathway thermodynamics, and a genetic algorithm for metabolic flux determination and optimal metabolic network design," BMC Systems Biology, vol. 4, article no. $49,2010$.

[51] S. Li, D. Huang, Y. Li, J. Wen, and X. Jia, "Rational improvement of the engineered isobutanol-producing Bacillus subtilis by elementary mode analysis," Microbial Cell Factories, vol. 11, article no. 101, 2012.

[52] F. Kracke and J. O. Krömer, "Identifying target processes for microbial electrosynthesis by elementary mode analysis," $B M C$ Bioinformatics, vol. 15, no. 1, article no. 410, 2014.

[53] G. Stephanopoulos, A. Aristidou, and J. Nielsen, Metabolic engineering: principles and methodologies, 1998.

[54] J. J. Heijnen, W. M. Van Gulik, H. Shimizu, and G. Stephanopoulos, "Metabolic flux control analysis of branch points: An improved approach to obtain flux control coefficients from large perturbation data," Metabolic Engineering, vol. 6, no. 4, pp. 391400, 2004.
[55] M. R. Antoniewicz, "Methods and advances in metabolic flux analysis: a mini-review," Journal of Industrial Microbiology and Biotechnology, vol. 42, no. 3, pp. 317-325, 2015.

[56] J. Niklas, K. Schneider, and E. Heinzle, "Metabolic flux analysis in eukaryotes," Current Opinion in Biotechnology, vol. 21, no. 1, pp. 63-69, 2010.

[57] L.-E. Quek, S. Dietmair, J. O. Krömer, and L. K. Nielsen, "Metabolic flux analysis in mammalian cell culture," Metabolic Engineering, vol. 12, no. 2, pp. 161-171, 2010.

[58] S. A. Rahman and D. Schomburg, "Observing local and global properties of metabolic pathways: 'Load points' and 'choke points' in the metabolic networks," Bioinformatics, vol. 22, no. 14, pp. 1767-1774, 2006.

[59] S. Mehta and S. Tagore, "Functional module analysis in metabolomics: chokes", Advances in Computational Research, vol. 1, no. 1, pp. 1-4, 2009.

[60] T. Y. Kim, H. U. Kim, and S. Y. Lee, "Metabolite-centric approaches for the discovery of antibacterials using genomescale metabolic networks," Metabolic Engineering, vol. 12, no. 2, pp. 105-111, 2010.

[61] C. S. Henry, L. J. Broadbelt, and V. Hatzimanikatis, “Thermodynamics-based metabolic flux analysis," Biophysical Journal, vol. 92, no. 5, pp. 1792-1805, 2007.

[62] S. Garg, L. Yang, and R. Mahadevan, “Thermodynamic analysis of regulation in metabolic networks using constraint-based modeling," BMC Research Notes, vol. 3, article no. 125, 2010.

[63] R. Moreno-Sánchez, E. Saavedra, S. Rodríguez-Enríquez, J. C. Gallardo-Pérez, H. Quezada, and H. V. Westerhoff, "Metabolic control analysis indicates a change of strategy in the treatment of cancer," Mitochondrion, vol. 10, no. 6, pp. 626-639, 2010.

[64] F. B. K. Özbayraktar and K. Ö. Ülgen, "Drug target identification in sphingolipid metabolism by computational systems biology tools: Metabolic control analysis and metabolic pathway analysis," Journal of Biomedical Informatics, vol. 43, no. 4, pp. 537-549, 2010.

[65] Z. González-Chávez, V. Olin-Sandoval, J. S. Rodíguez-Zavala, R. Moreno-Sánchez, and E. Saavedra, "Metabolic control analysis of the Trypanosoma cruzi peroxide detoxification pathway identifies tryparedoxin as a suitable drug target," Biochimica et Biophysica Acta (BBA) - General Subjects, vol. 1850, no. 2, pp. 263-273, 2015.

[66] E. O’Duibhir, P. Lijnzaad, J. J. Benschop et al., "Cell cycle population effects in perturbation studies," Molecular Systems Biology, vol. 10, no. 6, pp. 732-732, 2014.

[67] E. Szczurek and N. Beerenwinkel, "Linear effects models of signaling pathways from combinatorial perturbation data," Bioinformatics, vol. 32, no. 12, pp. i297-i305, 2016.

[68] R. Caspi, R. Billington, L. Ferrer et al., “The MetaCyc database of metabolic pathways and enzymes and the BioCyc collection of pathway/genome databases," Nucleic Acids Research, vol. 44, no. 1, pp. D471-D480, 2016.

[69] T. Kim, K. Dreher, R. Nilo-Poyanco et al., "Patterns of metabolite changes identified from large-scale gene perturbations in arabidopsis using a genome-scale metabolic network," Plant Physiology, vol. 167, no. 4, pp. 1685-1698, 2015.

[70] S. Bazzani, A. Hoppe, and H.-G. Holzhütter, "Network-based assessment of the selectivity of metabolic drug targets in Plasmodium falciparum with respect to human liver metabolism," BMC Systems Biology, vol. 6, article no. 118, 2012. 


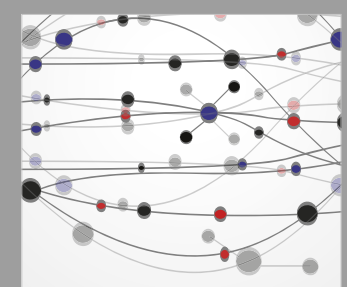

The Scientific World Journal
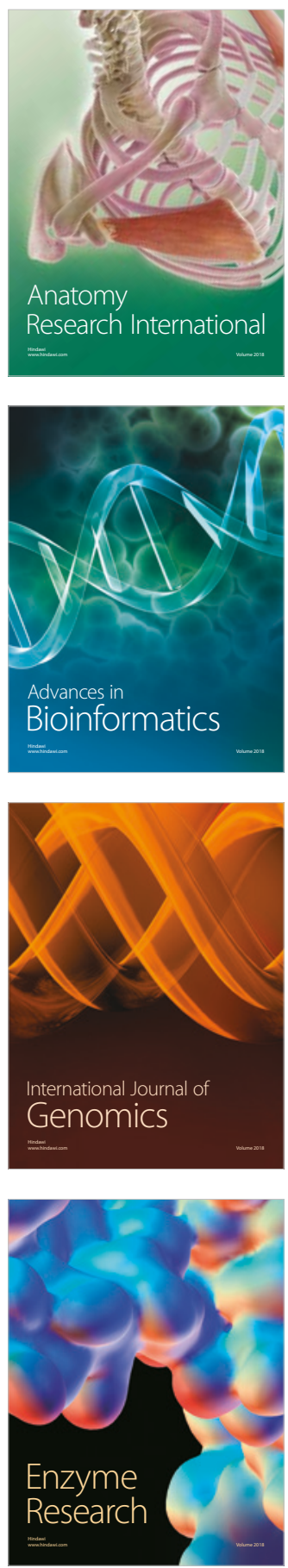
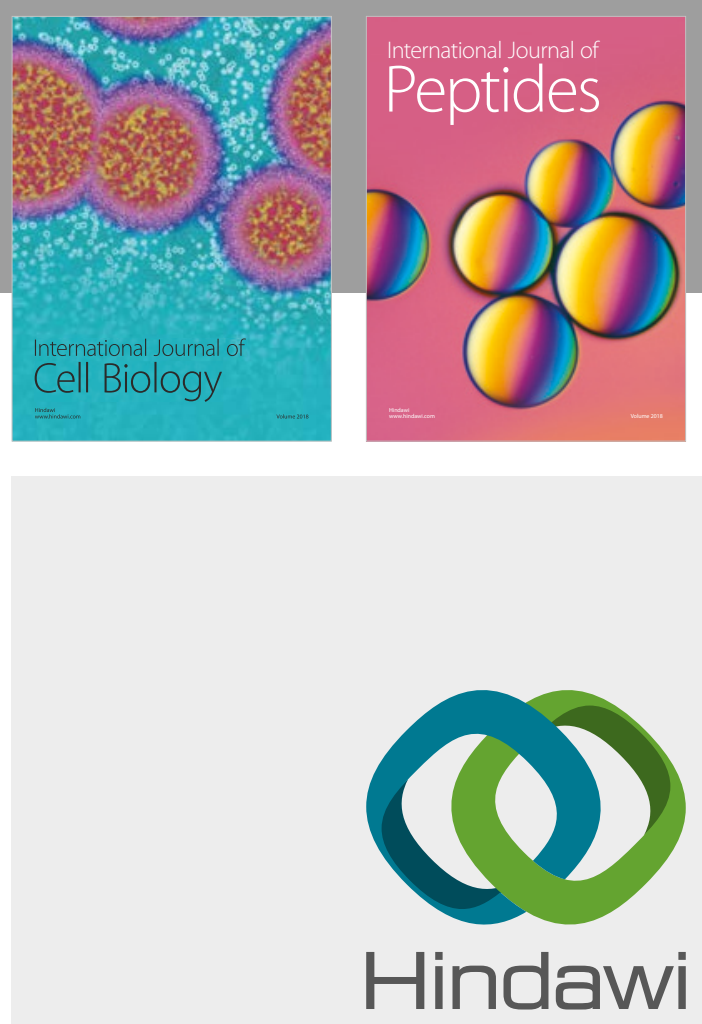

Submit your manuscripts at

www.hindawi.com
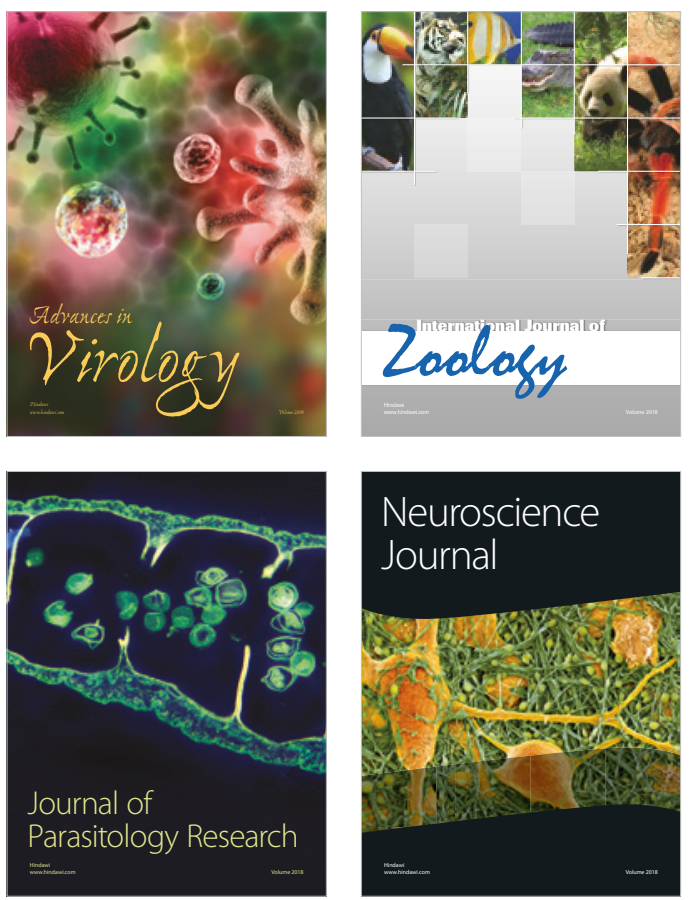
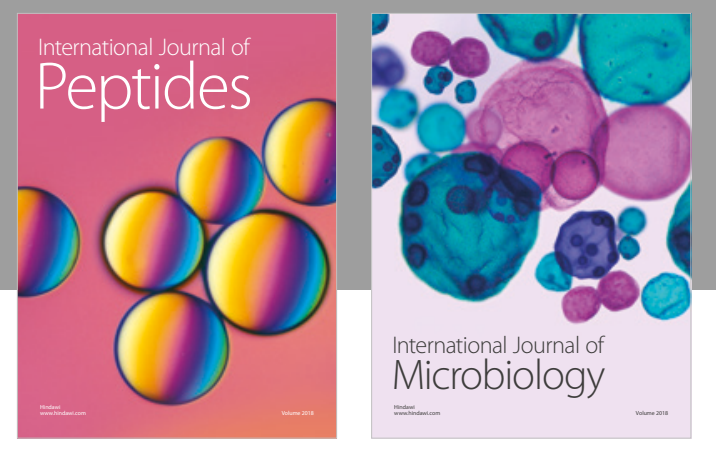

nternational Journal of Microbiology
Journal of
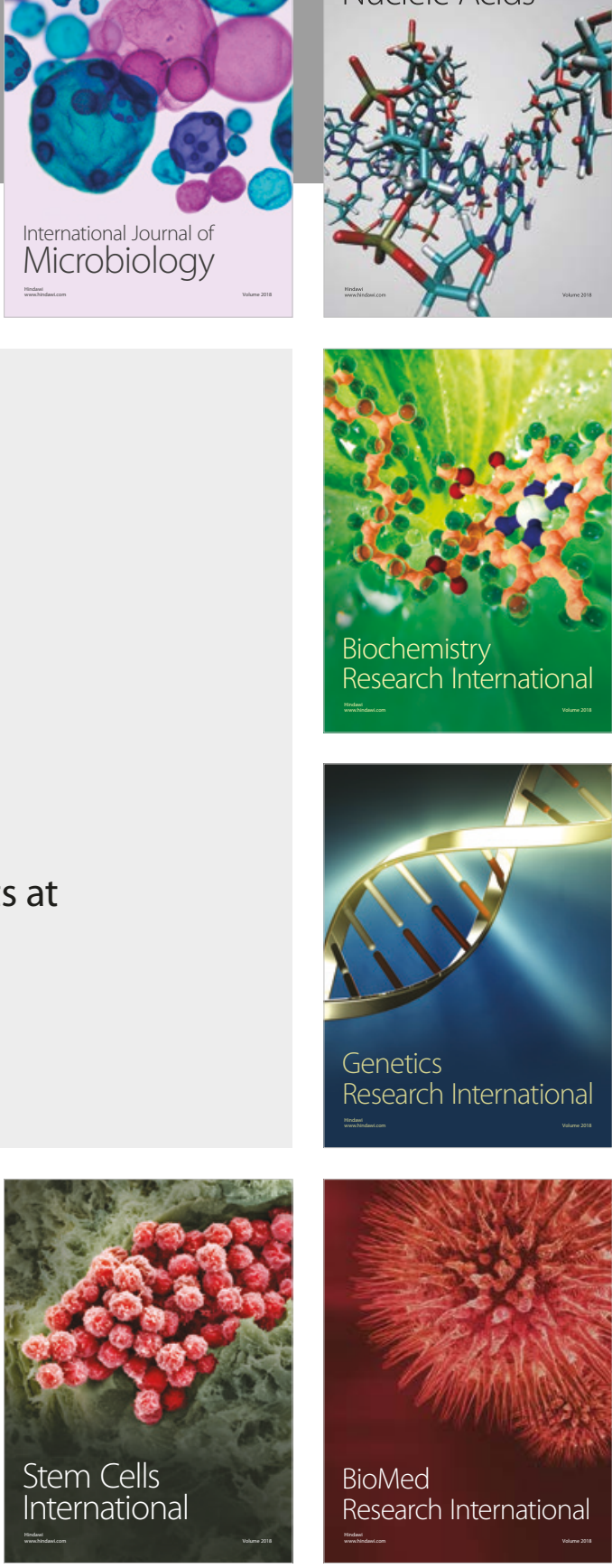
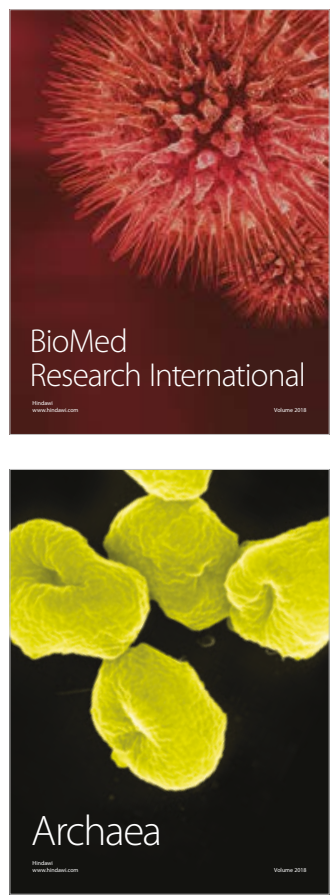